\title{
Predictors of Renal Replacement Therapy in Acute Kidney Injury
}

\author{
Michael J. Koziolek ${ }^{a}$ Rabi R. Datta ${ }^{a}$ Harry Mattes $^{\text {a }}$ \\ Klaus Jung ${ }^{b}$ Daniel Heise ${ }^{c}$ Jan H. Streich ${ }^{a}$ \\ Johannes Mühlhausen ${ }^{\mathrm{a}}$ Gerhard A. Mueller $^{\mathrm{a}}$ Hassan Dihazi $^{\mathrm{a}}$ \\ Departments of a Nephrology and Rheumatology, ${ }^{\mathrm{b}}$ Medical Statistics, and \\ 'Anaesthesiology, Georg August University Göttingen, Göttingen, Germany
}

\section{Key Words}

Acute kidney injury $\cdot$ Dialysis $\cdot$ Logistic regression model $\cdot$ Renal failure $\cdot$ Renal

replacement therapy

\begin{abstract}
Backgrounds: Criteria that may guide early renal replacement therapy (RRT) initiation in patients with acute kidney injury (AKI) currently do not exist. Methods: In 120 consecutive patients with AKI, clinical and laboratory data were analyzed on admittance. The prognostic power of those parameters which were significantly different between the two groups was analyzed by receiver operator characteristic curves and by leave-1-out cross validation. Results: Six parameters (urine albumin, plasma creatinine, blood urea nitrogen, daily urine output, fluid balance and plasma sodium) were combined in a logistic regression model that estimates the probability that a particular patient will need RRT. Additionally, a second model without daily urine output was established. Both models yielded a higher accuracy (89 and 88\% correct classification rate, respectively) than the best single parameter, cystatin C (correct classification rate $74 \%$ ). Conclusions: The combined models may help to better predict the necessity of RRT using clinical and routine laboratory data in patients with AKI.

Copyright $\odot 2012$ S. Karger AG, Basel
\end{abstract}




\section{Introduction}

The incidence of acute kidney injury (AKI) varies up to $30-50 \%$ of patients in intensive care units [1]. Its occurrence adversely affects the prognosis of patients [2]. The mortality of critically ill patients with severe AKI averages up to $61 \%$ [3].

The definition of AKI is contentious and over 35 different definitions have been used [4]. Recently, the Acute Dialysis Quality Initiative (ADQI) published a definition which provided a consensus and evidence-based definition for AKI [5]. The so-called RIFLE classification describes three grades of severity (Risk, Injury, Failure) and two clinical outcomes (Loss, End-stage). More recently, the Acute Kidney Injury Network (AKIN) supplemented the RIFLE criteria with some refinements [6]. Thus, AKI is defined as an abrupt reduction in kidney function within $48 \mathrm{~h}$, currently defined as an absolute increase or a percentage increase in serum creatinine, or a reduction in urine output.

Through these classifications, the definition of renal failure has become more precise, but the capability to handle it is still extremely limited. There are the conventional indications for initiating renal replacement therapy (RRT) [7], but the optimal timing is still not well defined. Early initiation of RRT is recommended because it may improve the clinical outcome of patients who develop AKI $[8,9]$, although this is still controversially discussed [10]. However, criteria that may guide an early initiation of therapy in patients with AKI do currently not exist $[11,12]$. There are only a few studies which investigated the optimal point of time to begin RRT. Some prior and a few recent retrospective studies separately suggest a positive impact of early RRT initiation on mortality [8, 9, 13-17].

Thus, the intention of this study was to evaluate clinical routine urine and blood parameters in order to predict the necessity for RRT.

\section{Patients and Methods}

\section{Patients}

Patients were recruited in two medical and four anesthesiological intensive care units (ICUs) as well as in one medical intermediate care (IMC) unit of the University of Göttingen, Germany. Patients were prospectively screened, and in case of a deterioration of kidney function (i.e. a serum creatinine rise of at least $0.3 \mathrm{mg} / \mathrm{dl}$ or $50 \%$ of base value, or a urine output of $<0.5 \mathrm{ml} / \mathrm{kg}$ body weight/h over $>6 \mathrm{~h}$ ), patients were included into the study. AKI was detected either on admission or in the course of hospitalization. Patients with a previous serum creatinine $\geq 2.5 \mathrm{mg} / \mathrm{dl}$ were excluded.

Clinical sample acquisition and analyses as well as data management of this study were approved by the local Ethics Committee of the University Hospital of the Georg August University, Göttingen, Germany. All patients or their legal guardian gave their written informed consent prior to their study inclusion.

Biometric data (gender, age, size, weight and body mass index), relevant past history (diabetes mellitus, coronary heart disease, chronic obstructive lung disease, occlusive artery disease, congestive heart failure and chronic renal failure stage II or III), study-relevant clinical data (daily urine output, fluid balance) and laboratory values from the day of admittance were evaluated.

\section{Clinical Chemistry}

Besides standard blood parameters (blood count, creatinine, blood urea nitrogen (BUN), C-reactive protein and albumin), creatinine, protein, sodium, potassium, urea nitrogen, osmolarity, albumin and $\alpha 1$-microglobuline concentrations were analyzed in urine samples 
with standard routine methods at the Department of Clinical Chemistry or the laboratory of the Department of Nephrology and Rheumatology, Georg August University Göttingen, Germany. The $\mathrm{pH}$ value was routinely determined by arterial or venous blood gas analysis depending on the clinical situation. Due to different sample origin, the data were not used for statistical comparison. Additionally, follow-up data were obtained for the parameters plasma creatinine, fluid balance and daily urine output.

Moreover, some additional parameters were measured according to the manufacturer's protocol in either serum or plasma using commercial test kits to further define the type of AKI: complement factors $\mathrm{C} 3 \mathrm{c}$ and $\mathrm{C} 4, \mathrm{C}$-reactive protein, cystatin $\mathrm{C}$ in serum, $\alpha 1$-microglobulin and albumin in urine (all six Dade Behring, Schwallbach, Germany), anti-neutrophil cytoplasmic antibody and anti-nuclear antibody (both Inova Diagnostics, San Diego, Calif. USA), anti-glomerular basement membrane antibodies (Phadia AB, Uppsala, Sweden) and circulating immune complexes (Phadia AB, Uppsala, Sweden), interleukin-1 $\beta$ and -6 , and tumor necrosis factor- $\alpha$ (Dade Behring, Schwallbach, Germany).

\section{Dialysis}

Depending on the necessity for RRT in the follow-up, patients were divided into two different groups (dialysis vs. non-dialysis). RRT was initiated by the attending physician based on laboratory data and clinical judgment. In this regard, RRT initiation was geared to current undisputed RRT indications such as persistent hyperkalemia, uremic serositis, encephalopathy, hypervolemia and acidosis which were refractory to conservative treatment [18]. In addition, renal parameters like BUN, creatinine and fluid balance were taken into account, although no clear-cut criteria were determined [19]. Dialysis modalities were chosen individually including intermittent or continuous hemodialysis modalities.

\section{Statistical Methods}

Distributions of categorical patient characteristics and diseases were compared between the dialyzed and the non-dialyzed group using $\chi^{2}$ tests, whereas continuous parameters were either compared by $t$ tests or by Wilcoxon rank-sum tests. The normality assumption for those parameters which were analyzed by the $t$ test was checked by quantile-quantile plots.

The prognostic power of those parameters which were significantly different between the two groups was analyzed by receiver operator characteristic (ROC) curves and by leave1 -out cross validation. In all ROC curve analyses, sensitivity, specificity and accuracy (given by the area under the curve, AUC) of the parameters or the regression model were studied. The AUC is an estimate for the potential accuracy of a parameter or a parameter combination. The accuracy gives the portion of correct classified individuals. In order to find a better classification rule, the most promising parameters were combined using logistic regression and stepwise variable selection. Missing values in the multivariate data matrix (approximately 6\%) were imputed by row means. The combined classification rule was again evaluated by a ROC curve analysis.

All tests were conducted with a significance level of $\alpha=0.05$. Calculations were all performed with the free software R 2.6 (www.r-project.org).

\section{Results}

\section{Patients}

Within 6 months, 564 patients had been screened for AKI, 120 patients were recruited into the study and 4 patients were excluded depending on whether they fulfilled AKIN criteria $[7,9]$ or not. Fifty-two patients had to undergo RRT (dialysis group) during the clinical 
Fig. 1. Screening, follow-up, assignment, updating data and selection for statistical analysis of AKI patients. * Screening on ICU/IMC admission. ** Anamnestic serum creatinine $\geq 2.5$ $\mathrm{mg} / \mathrm{dl}$.

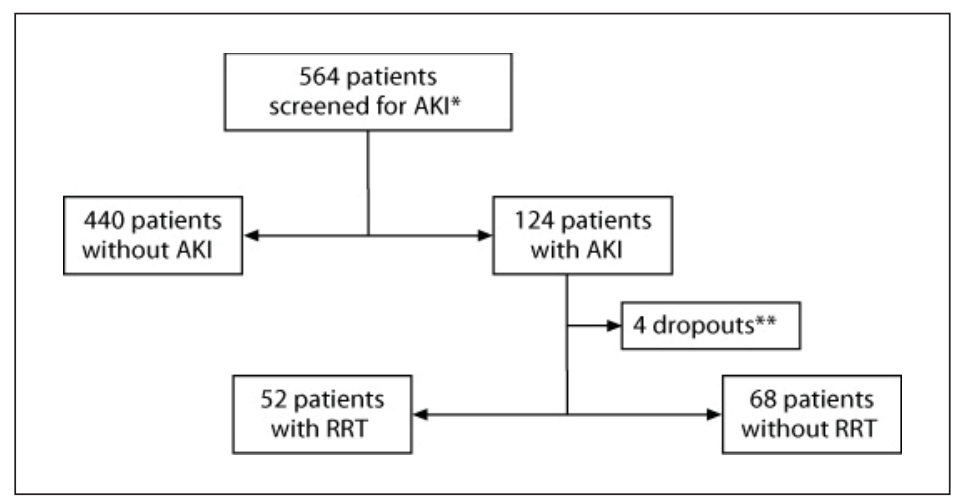

course and 68 did not (non-dialysis group) (fig. 1). Both groups were comparable with regard to patients' characteristics (table 1).

\section{Types of Renal Failure and Premorbid Diseases}

Dialyzed patients had higher AKIN scores. Origin (prerenal, intrarenal, postrenal) of AKI did not differ between the two different groups. When prerenal AKI was divided into subgroups, significantly more patients of the dialysis group suffered from septic shock; vice versa, cardiac shock was more frequent in the non-dialysis group. Consistently, non-dialysis patients suffered more often from coronary heart disease. The SAPS-II score tended to be higher in dialysis versus non-dialysis patients (median 34 vs. 28). Other types of premorbid diseases did not differ between the two groups (table 1).

\section{Blood and Urine Parameters}

On admittance, the daily urine output was significantly higher in the non-dialysis group (median 725 vs. $2,760 \mathrm{ml} /$ days), independent of diuretics use, and fluid balance (daily fluid input minus urine output) was higher in the dialysis group $(1,180.0 \pm 280.3 \mathrm{vs} .335 .0 \pm 194.9$ $\mathrm{ml})$. In blood samples, cystatin C ( $3.0 \pm 0.2 \mathrm{vs} .2 .1 \pm 0.1 \mathrm{mg} / \mathrm{l})$, plasma creatinine $(2.9 \pm 0.2$ vs. $2.1 \pm 0.1 \mathrm{mg} / \mathrm{dl}$ ) and BUN (median $23.4 \mathrm{vs} .16 .8 \mathrm{mg} / \mathrm{dl}$ ) were significantly higher in the dialysis group than in the non-dialysis group. Plasma $\mathrm{Na}(\mathrm{P}-\mathrm{Na})$ in the non-dialysis group (median 142.5 (range 128-159) vs. 140.0 (range 108-154) mmol/l) was higher than in the dialysis group. In urine samples, albuminuria (median 70.5 (range 13-3,200) vs. 34.0 (range $12-681) \mathrm{mg} / \mathrm{l}), \mathrm{Na} / \mathrm{creatinine}$ ratio $(3.1 \pm 0.3 \mathrm{vs} .4 .3 \pm 0.3 \mathrm{mmol} / \mathrm{mg}$ ) and $\mathrm{K} /$ creatinine ratio (median 1.7 (range $0.3-5.7$ ) vs. 2.1 (range $0.3-6.5$ ) $\mathrm{mmol} / \mathrm{mg}$ ) were significantly higher in the non-dialysis group. Results are summarized in table 2 . There was no difference between the two groups concerning the other determined laboratory values (data not shown).

During the study, the maximum creatinine (median 3.6 vs. $2.4 \mathrm{mg} / \mathrm{dl}$ ) and creatinine increase (median 3.6 vs. $2.9 \mathrm{mg} / \mathrm{dl}$ ) was significantly higher in the dialysis group (table 3 ).

\section{Logistic Regression Model}

The ability of selected parameters on ICU/IMC admission to classify patients into the dialysis or the non-dialysis group is illustrated in table 4 . Ten of these parameters showed an accuracy better than a random assignment (confidence intervals of their AUCs did not include the $50 \%$ accuracy). Serum albumin, cystatin C, plasma creatinine, P-Na, BUN, proteinuria, urine $\mathrm{Na}(\mathrm{U}-\mathrm{Na})$, urine $\mathrm{K}(\mathrm{U}-\mathrm{K})$, fluid balance and daily urine output were combined in a logistic regression model where proteinuria, cystatin $\mathrm{C}, \mathrm{U}-\mathrm{Na}$ and $\mathrm{U}-\mathrm{K}$ were removed by stepwise variable selection. This yielded the following classification rule: probability (person 
Table 1. Patients' characteristics, disease scores, disease classifications, SAPS II score and relevant premorbidities

\begin{tabular}{|c|c|c|c|}
\hline & \multicolumn{2}{|l|}{ Group } & \multirow[t]{2}{*}{$\mathrm{p}$} \\
\hline & $\begin{array}{l}\text { dialysis } \\
(\mathrm{n}=52)\end{array}$ & $\begin{array}{l}\text { non-dialysis } \\
(\mathrm{n}=68)\end{array}$ & \\
\hline \multicolumn{3}{|l|}{ Gender } & 0.8285 \\
\hline Male & $34(65)$ & $42(62)$ & \\
\hline Female & $18(35)$ & $26(38)$ & \\
\hline Age, years & $68.2 \pm 2.1$ & $70.3 \pm 1.5$ & 0.4040 \\
\hline Height, cm & $170.3 \pm 1.3$ & $170.8 \pm 1.1$ & 0.7868 \\
\hline Weight, kg & $79.9 \pm 2.9$ & $81.4 \pm 2.2$ & 0.6791 \\
\hline Body mass index & $27.5 \pm 1.1$ & $28.0 \pm 0.7$ & 0.7403 \\
\hline \multicolumn{3}{|l|}{ AKIN score } & $<2.2 \times 10^{-16}$ \\
\hline 1 & 0 & $39(57.4)$ & \\
\hline 2 & 0 & $20(29.4)$ & \\
\hline 3 & $52(100)$ & $9(13.2)$ & \\
\hline \multicolumn{3}{|l|}{ Type of AKI } & 0.1836 \\
\hline Intrarenal & $3(5.8)$ & 0 & \\
\hline Prerenal & $48(92.3)$ & $65(95.6)$ & \\
\hline Postrenal & 0 & 0 & \\
\hline Others $^{1}$ & $1(1.9)$ & $2(2.9)$ & \\
\hline Unknown $^{2}$ & 0 & $1(1.5)$ & \\
\hline \multicolumn{3}{|l|}{ Subtypes of prerenal AKI } & $7.464 \times 10^{-5}$ \\
\hline Hypovolemic & $1(2.1)$ & $3(4.6)$ & \\
\hline Cardiac & $17(35.4)$ & $48(73.8)$ & \\
\hline Septic & $24(50.0)$ & $8(12.3)$ & \\
\hline Other & $6(12.5)$ & $6(9.2)$ & \\
\hline SAPS II score (range) & $34(9-72)$ & $28(6-58)$ & 0.09613 \\
\hline \multicolumn{4}{|l|}{ Premorbid diseases } \\
\hline Coronary heart disease & $24(47.1)$ & $48(72.7)$ & 0.008332 \\
\hline
\end{tabular}

Patients' characteristics are either described by absolute and relative proportions (numbers with percentages in parentheses) or by means $\pm \mathrm{SE}$, unless otherwise indicated. ${ }^{1}$ Combination of pre-, intra- and/ or postrenal AKI. ${ }^{2}$ In 1 patient, origin of AKI was left unknown.

in dialysis group $)=\exp (\mathrm{x}) /(1+\exp (\mathrm{x}))$, where $\mathrm{x}=10.1+0.005 \times$ urine albumin $+0.59 \times$ plasma creatinine $+0.06 \times$ BUN $-0.0003 \times$ daily urine output $+0.0008 \times$ fluid balance $-0.098 \times \mathrm{P}-\mathrm{Na}$. As optimal cutoff for this probability equation, 0.61 was determined.

Additionally, a further model was established without daily urine output, which (after variable selection) yielded the following coefficients: $\mathrm{x}=7.36+0.005 \times$ urine albumin +0.59 $\times$ plasma creatinine $+0.05 \times \mathrm{BUN}+0.0009 \times$ fluid balance $-0.086 \times \mathrm{P}-\mathrm{Na}$. In a comparison of both models, the loss of accuracy is very small when not considering daily urine output.

Altogether, the combination without daily urine output yielded a better accuracy than the best single parameter, cystatin C (table 4). In ROC curve analysis of the combination without daily urine output (fig. 2), the optimal cutoff to distinguish between the necessity of RRT was 0.48 giving a sensitivity of $77 \%$ and a specificity of $82 \%$, respectively, with an accuracy of $88 \%$. Using these formulas, the necessity of RRT was predictable with a mean of 3.6 days (median 1 (range $0-24$ ) days) before initiation. 


Nephron Extra 2012;2:247-255
\begin{tabular}{l|l}
\hline DOI: $10.1159 / 000342257$ \\
Published online: September 21, 2012 & $\begin{array}{l}\text { @ } 2012 \text { S. Karger AG, Basel } \\
\text { www.karger.com/nne }\end{array}$ \\
\hline
\end{tabular}

Koziolek et al.: Predictors of Renal Replacement Therapy in Acute Kidney Injury

Table 2. Summary of plasma markers, clinical findings, urine parameters and immunological markers with significant differences between the group of dialyzed and non-dialyzed patients on admittance

\begin{tabular}{|c|c|c|c|c|c|}
\hline \multirow[t]{2}{*}{ Parameter } & \multicolumn{4}{|l|}{ Group } & \multirow[t]{2}{*}{$\mathrm{p}$} \\
\hline & \multicolumn{2}{|c|}{ dialysis $(n=52)$} & \multicolumn{2}{|c|}{ non-dialysis $(\mathrm{n}=68)$} & \\
\hline S-cystatin C, mg/l & $(\mathrm{n}=48)$ & $3.0 \pm 0.2$ & $(n=66)$ & $2.1 \pm 0.1$ & $9.796 \times 10^{-6}$ \\
\hline P-creatinine, $\mathrm{mg} / \mathrm{dl}$ & $(\mathrm{n}=52)$ & $2.9 \pm 0.2$ & $(n=68)$ & $2.1 \pm 0.1$ & 0.0005458 \\
\hline P-BUN, mg/dl & $(\mathrm{n}=51)$ & $23.4(8.4-68.2)$ & $(n=65)$ & $16.8(8.4-57.4)$ & 0.0002563 \\
\hline $\mathrm{P}-\mathrm{Na}, \mathrm{mmol} / \mathrm{l}$ & $(\mathrm{n}=48)$ & $140.0(108.0-154.0)$ & $(n=62)$ & $142.5(128.0-159.0)$ & 0.01915 \\
\hline Daily urine output, $\mathrm{ml}$ & $(\mathrm{n}=45)$ & $725(0.0-6,790)$ & $(n=65)$ & $2,760(90-8,400)$ & $1.966 \times 10^{-5}$ \\
\hline Fluid balance & $(\mathrm{n}=47)$ & $1,180.0 \pm 280.3$ & $(n=65)$ & $335.0 \pm 194.9$ & 0.01524 \\
\hline \multicolumn{6}{|l|}{ U-potassium/creatinine } \\
\hline $\mathrm{mmol} / \mathrm{mg}$ & $(n=46)$ & $1.7(0.3-5.7)$ & $(\mathrm{n}=68)$ & $2.1(0.3-6.5)$ & 0.04536 \\
\hline $\mathrm{U}-\mathrm{Na} /$ creatinine, $\mathrm{mmol} / \mathrm{mg}$ & $(n=43)$ & $3.1 \pm 0.3$ & $(n=68)$ & $4.3 \pm 0.3$ & 0.003105 \\
\hline U-albumin, mg/l & $(\mathrm{n}=44)$ & $70.5(13.0-3,200.0)$ & $(\mathrm{n}=47)$ & $34.0(12.0-681.0)$ & 0.0004598 \\
\hline
\end{tabular}

Values are available sample sizes with means \pm SE or medians with ranges. S- = Serum; P- = plasma; $\mathrm{U}$ - = urine.

Table 3. Comparison of baseline and maximal creatinine as well as creatinine increase (either as 48-hour value or maximal increase) between the group of dialyzed and non-dialyzed patients

\begin{tabular}{|c|c|c|c|}
\hline \multirow[t]{2}{*}{ Parameter } & \multicolumn{2}{|l|}{ Group } & \multirow[t]{2}{*}{$\mathrm{p}$} \\
\hline & dialysis $(\mathrm{n}=52)$ & non-dialysis $(\mathrm{n}=68)$ & \\
\hline Baseline creatinine, mg/dl & $(\mathrm{n}=51) \quad 1.0(0.5-2.2)$ & $(\mathrm{n}=68) \quad 0.9(0.5-1.0)$ & 0.01711 \\
\hline Maximum creatinine, $\mathrm{mg} / \mathrm{dl}$ & $(\mathrm{n}=52) \quad 3.6(1.2-4.6)$ & $(\mathrm{n}=68) \quad 2.4(1.1-2.8)$ & $3.55 \times 10^{-9}$ \\
\hline Creatinine increase within $48 \mathrm{~h}, \mathrm{mg} / \mathrm{dl}$ & $(\mathrm{n}=38) \quad 1.6(0.0-4.8)$ & $(\mathrm{n}=66) \quad 1.8(1.0-3.5)$ & 0.08466 \\
\hline Maximum creatinine increase, $\mathrm{mg} / \mathrm{dl}$ & $(\mathrm{n}=48) \quad 3.6(0.0-12.4)$ & $(\mathrm{n}=68) 2.9(1.3-3.4)$ & 0.001327 \\
\hline
\end{tabular}

Values are available sample sizes and median (min-max).

\section{Discussion}

Despite improvements in intensive care medicine and RRT, AKI in the critically ill patient is still associated with a high mortality, especially when RRT is required [20]. Among those patients, initiation of dialysis at higher BUN concentrations was associated with an increased risk for death [21]. An implication of these data is that optimization of renal support may reduce its high mortality. Although multiple recent clinical trials have prospectively evaluated the impact of dose and modality of RRT, the literature on timing of RRT in AKI is far less robust [9]. During the past decade, in most studies concerning timing and mortality, RRT initiation was based on the findings of only 1-3 biomarkers of renal function, i.e. solely BUN, potassium, serum creatinine or daily diuresis [9].

Since the major renal functions are excreting uremic toxins and maintaining volume, electrolyte, and acid-base homeostasis, the failure of these functions can lead to urgent, commonly undoubted, indications for RRT [19]. A specific BUN or serum creatinine concentration at which start of RRT is indicated in AKI patients is difficult to define and, thus, a clear cutoff was excluded in our study. Most cases of ICU-associated AKI occur under non-steady- 
Table 4. Characterization of selected parameters with regard to their ability for classifying dialyzed patients

\begin{tabular}{llllc}
\hline Parameter & $\begin{array}{l}\text { Sensitivity } \\
\%\end{array}$ & $\begin{array}{l}\text { Specificity } \\
\%\end{array}$ & $\begin{array}{l}\text { AUC, \% } \\
(95 \% \mathrm{CI})\end{array}$ & $\begin{array}{l}\text { Optimal cutoff } \\
\text { mean } \pm \text { SE }\end{array}$ \\
\hline Cystatin C, mg/l & 71 & 65 & 74 & $2.4 \pm 0.0006$ \\
P-creatinine, mg/dl & 42 & 78 & 70 & $2.8 \pm 0.01$ \\
BUN, mg/dl & 42 & 81 & $70(60-79)$ & $23.1 \pm 0.08$ \\
P-Na, mmol/l & 40 & 58 & 63 & $141.9 \pm 0.05$ \\
U-protein/creatinine, mg/mg & 56 & 65 & 60 & $17.3 \pm 0.002$ \\
U-Na/creatinine, mmol/mg & 78 & 51 & $70(60-81)$ & $2.5 \pm 0.007$ \\
U-potassium/creatinine, mmol/mg & 32 & 91 & $61(51-72)$ & $3.0 \pm 0.0009$ \\
U-albumin, mg/l & 61 & 62 & 71 & $63.2 \pm 0.7$ \\
Fluid balance & 71 & 50 & $64(51-77)$ & $1,218.1 \pm 1.2$ \\
Daily urine output, ml/day $_{\text {Combination }}^{1}$ & 78 & 70 & $73(64-84)$ & $1,689.7 \pm 0.3$ \\
Combination without daily urine output $^{1}$ & 77 & 83 & $89(83-95)$ & $0.53 \pm 0.0004$ \\
\end{tabular}

P- = Plasma; $\mathrm{U}$ - = urine.${ }^{1}$ Logistic regression model including urine albumin, plasma creatinine, BUN, daily urine output, fluid balance and plasma sodium.

Fig. 2. ROC curve analysis. Combination A: logistic regression model including urine albumin, plasma creatinine, BUN, daily urine output, fluid balance and plasma sodium. Combination B: without daily urine output.

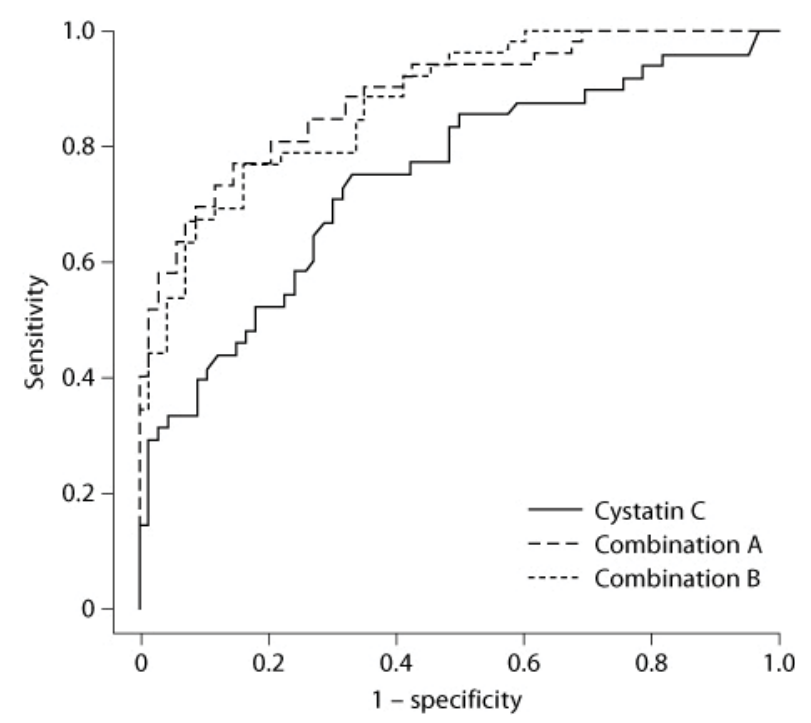

state conditions in which the determinants of serum creatinine and BUN concentration fluctuate. Likewise, several individual variables, such as metabolic status, medication, muscle mass or age, have an impact on serum creatinine or BUN levels. Therefore, daily changes in serum creatinine or BUN concentration poorly reflect the actual glomerular filtration rate $[19,22]$. Moreover, there is still no clear agreement about optimal cutoff values for RRT initiation regarding the above-mentioned biochemical parameters or clinical circumstances [7, 10]. In summary, there is only little consensus on the proper time to initiate RRT in patients 
with AKI or, vice versa, a strong need for suitable parameters which help physicians to decide when to start RRT. The expectations concerning such parameters are high accuracy and ubiquitous availability combined with low costs.

In our prospective study, we could show that with routinely analyzed parameters it is possible to distinguish between patients presumably requiring RRT or not with an accuracy of $88 \%$. These parameters included laboratory as well as clinical data normally impaired by AKI, i.e. albuminuria, plasma creatinine and sodium, BUN and fluid balance. Our statistical models yielded a higher accuracy than the best single parameter, cystatin C. In recent years, there have been several studies on novel biomarkers for early diagnosis of AKI. The existing panel of emerging biomarkers currently still displays some limitations, having been validated mostly in animal models of AKI or in a limited number of clinical studies. If low urine output exists, the validity of a small urine sample taken regarding the levels of distinct biomarkers remains questionable [23]. We, therefore, excluded novel biomarkers from our study.

In one of our regression models, the daily urine output has been excluded due to the hypothesis that the measurement of urinary excretion potentially lacks accuracy, especially on the first day of admission. This circumstance results from various procedures patients go through and, consequently, absence of or change in attending personnel. In addition, daily urine output was excluded due to variations of diuretic dosage among patients. Moreover, the loss of accuracy was very small when not considering daily urine output. As these abovementioned parameters are determined routinely in AKI, no additional costs are necessary.

However, our study was limited by several factors. In clinical practice of ICUs, $\mathrm{pH}$ measurement was made from either arterial or venous blood samples. Due to incomparability of arterial versus venous $\mathrm{pH}$ values, we decided to exclude $\mathrm{pH}$ values from our analysis. Another limitation is that RRT was initiated on the attending clinician's decision based on clinical data and laboratory values. However, the clinical approach is in fact not only a simple reproduction of clinical findings, but rather a multi-input approach connecting physicians' experience, patients' history, laboratory parameters and data from routinely used techniques. Defining clear-cut values for RRT initiation would lead to exactly these values as thresholds in our statistical models and thereby devaluate them. Furthermore, we did not define clearcut values since an optimal time or situation to start RRT in AKI treatment has not been clearly defined yet [19]. With our analysis, we approached this problem and defined statistical models which give us an accurate prediction of the probability of RRT necessity on the first day of ICU/IMC admission with a mean of 3.6 days prior to its initiation.

\section{Conclusions}

In conclusion, despite conventionally accepted indications for initiation of RRT, the optimal timing for initiation of RRT in patients with AKI remains uncertain. Our statistical model includes several routine laboratory and clinical parameters that may help to create criteria for RRT initiation.

\section{Disclosure Statement}

The authors have no conflicts of interest. 


\section{References}

-1 Xue JL, Daniels F, Star RA, Kimmel PL, Eggers PW, Molitoris BA, Himmelfarb J, Collins AJ: Incidence and mortality of acute renal failure in Medicare beneficiaries, 1992 to 2001. J Am Soc Nephrol 2006;17:1135-1142.

-2 Novis BK, Roizen MF, Aronson S, Thisted RA: Association of preoperative risk factors with postoperative acute renal failure. Anesth Analg 1994;78:143-149.

-3 Ostermann ME, Chang RW: Prognosis of acute renal failure: an evaluation of proposed consensus criteria. Intensive Care Med 2005;31:250-256.

4 Kellum JA, Levin N, Bouman C, Lameire N: Developing a consensus classification system for acute renal failure. Curr Opin Crit Care 2002;8:509-514.

-5 Bellomo R, Ronco C, Kellum JA, Mehta RL, Palevsky P: Acute renal failure - definition, outcome measures, animal models, fluid therapy and information technology needs: the Second International Consensus Conference of the Acute Dialysis Quality Initiative (ADQI) Group. Crit Care 2004; 8:R204-R212.

-6 Mehta RL, Kellum JA, Shah SV, Molitoris BA, Ronco C, Warnock DG, Levin A, Acute Kidney Injury Network: Report of an initiative to improve outcomes in acute kidney injury. Crit Care 2007;11:R31.

-7 Palevsky PM: Indications and timing of renal replacement therapy in acute kidney injury. Crit Care Med 2008;36:S224-S228.

8 Gettings LG, Reynolds HN, Scalea T: Outcome in post-traumatic acute renal failure when continuous renal replacement therapy is applied early vs. late. Intensive Care Med 1999;25:805-813.

-9 Palevsky PM: Clinical review: timing and dose of continuous renal replacement therapy in acute kidney injury. Crit Care 2007;11:232.

-10 Gibney N, Hoste E, Burdmann EA, Bunchman T, Kher V, Viswanathan R, Mehta RL, Ronco C: Timing of initiation and discontinuation of renal replacement therapy in AKI: unanswered key questions. Clin J Am Soc Nephrol 2008;3:876-880.

-11 Bellomo R, Ronco C: Indications and criteria for initiating renal replacement therapy in the intensive care unit. Kidney Int Suppl 1998;66:S106-S109.

-12 Bellomo R, Kellum J, Ronco C: Acute renal failure: time for consensus. Intensive Care Med 2001;27: $1685-1688$.

-13 Parsons FM, Hobson SM, Blagg CR, Mc CB: Optimum time for dialysis in acute reversible renal failure. Description and value of an improved dialyser with large surface area. Lancet 1961;1:129-134.

-14 Kleinknecht D, Jungers P, Chanard J, Barbanel C, Ganeval D, Rondon-Nucete M: Factors influencing immediate prognosis in acute renal failure, with special reference to prophylactic hemodialysis. Adv Nephrol Necker Hosp 1971;1:207-230.

-15 Conger JD: A controlled evaluation of prophylactic dialysis in post-traumatic acute renal failure. J Trauma 1975;15:1056-1063.

-16 Demirkilic U, Kuralay E, Yenicesu M, Caglar K, Oz BS, Cingoz F, Gunay C, Yildirim V, Ceylan S, Arslan M, Vural A, Tatar H: Timing of replacement therapy for acute renal failure after cardiac surgery. J Card Surg 2004;19:17-20.

-17 Piccinni P, Dan M, Barbacini S, Carraro R, Lieta E, Marafon S, Zamperetti N, Brendolan A, D’Intini V, Tetta C, Bellomo R, Ronco C: Early isovolaemic haemofiltration in oliguric patients with septic shock. Intensive Care Med 2006;32:80-86.

-18 Seabra VF, Balk EM, Liangos O, Sosa MA, Cendoroglo M, Jaber BL: Timing of renal replacement therapy initiation in acute renal failure: a meta-analysis. Am J Kidney Dis 2008;52:272-284.

-19 John S, Eckardt KU: Renal replacement strategies in the ICU. Chest 2007;132:1379-1388.

- 20 Clermont G, Acker CG, Angus DC, Sirio CA, Pinsky MR, Johnson JP: Renal failure in the ICU: comparison of the impact of acute renal failure and end-stage renal disease on ICU outcomes. Kidney Int 2002;62:986-996.

-21 Liu KD, Himmelfarb J, Paganini E, Ikizler TA, Soroko SH, Mehta RL, Chertow GM: Timing of initiation of dialysis in critically ill patients with acute kidney injury. J Am Soc Nephrol 2006;1:915-919.

-22 Moran M, Meyers BD: Cause of acute renal failure studied by a model of creatinine kinetics. Kidney Int 1985;27:928-933.

-23 Urbschat A, Obermüller N, Haferkamp A: Biomarkers of kidney injury. Biomarkers 2011;16:S22-S30. 